\title{
Hematomas e Infartos
} Cerebelares: Diagnóstico e Conduta

\author{
Maramélia Alves de Araújo Silva* \\ Marcia Maiumi Fukujima**
}

\section{RESUMO}

Mesmo com todo o avanço nos estudos de fisiopatologia, correlação anatomopatológica e técnicas de imagem, ainda não há ensaios clínicos suficientes a respeito da melhor terapêutica para o acometimento vascular cerebelar. As recomendações para o manejo dos pacientes seguem experiências restritas, notadamente em trabalhos retrospectivos. As séries apresentadas são pequenas, as controvérsias em relação à intervenção cirúrgica e ao tratamento clínico permanecem.

\section{UNITERMOS}

Infarto cerebelar, hemorragia cerebelar, diagnóstico, tratamento.
* Neurologista, em especialização no Setor de Neurorradiologia do Departamento de Diagnóstico por Imagem da Escola Paulista de Medicina - Unifesp.

** Neurologista do Setor de Doenças Neurovasculares da Escola Paulista de Medicina - Unifesp.

\section{NTRODUÇÃO}

As doenças vasculares que acometem o território posterior da circulação encefálica (território vertebrobasilar) têm sido menos estudadas do que as que envolvem o território anterior (carotídeo), porém não são de menor importância clínica. Costumam ser relacionadas a piores prognósticos e a evoluções fatais freqüentes. Recentemente, com a melhora das técnicas de imagens neurovasculares para diagnóstico precoce, associada a melhores condições de atendimento hospitalar, os hematomas e os infartos cerebelares têm recebido uma abordagem mais intensiva. Os hematomas cerebelares representam cerca de $10 \%$ das hemorragias intracranianas não-traumáticas.

Os hematomas e os infartos cerebelares, apesar de terem fisiopatologia bem distinta, comportam-se clinicamente com os mesmos sintomas e têm o manejo clínico-cirúrgico semelhante, pela sua alta probabilidade de evolução fatal, secundária a efeito compressivo na mesma topografia, fossa posterior, quer pelo hematoma, quer pelo edema do parênquima infartado sobre o tronco cerebral e sobre as estruturas adjacentes.

\section{QUADRO CLÍ NICO}

O quadro clínico deve-se à localização da lesão e ao seu efeito compressivo gradual sobre as estruturas da fossa posterior, notadamente o IV ventrículo e o tronco cerebral. Os sintomas como tontura, vertigem, ataxia, nistagmo, disartria, náuseas, vômitos e dismetria são os mais comuns nesses casos; alterações decorrentes do acometimento do tronco cerebral, como síndrome de Horner, síndrome de Wallemberg, hipoestesias, paresia facial, disfagia, zumbido ou alterações auditivas, são vistas em menor proporção de casos.

São descritas três fases de comprometimento neurológico no acidente vascular encefálico (AVE) do cerebelo (Quadro 1): 
QUADRO 1

Estágios do hematoma/infarto cerebelar

Estágio inicial -Sintomas cerebelovestibulares

Cefaléia

Insidiosos nos infartos

Súbitos nos hematomas

Estágio intermediário -Depressão do nível de consciência Início de HIC

Estágio tardio -Coma

Compressão do tronco cerebral

Alterações cardiovasculares

- A fase inicial deve-se à destruição do parênquima cerebelar e/ou à extensão do hematoma dentro do parênquima ou do espaço subaracnóideo; o paciente apresenta sintomas como tonturas, náuseas, vômitos, ataxia de marcha, cefaléia e desequilíbrio ${ }^{1}$. Esses sintomas tendem a ser mais abruptos nas hemorragias cerebelares e de instalação mais insidiosa nos infartos, e, nessa fase, os pacientes costumam estar com o nível de consciência preservado.

- A fase intermediária caracteriza-se por início ou aumento do efeito de massa, decorrente da lesão instalada anteriormente, quer por compressão do IV ventrículo, por aumento da hemorragia ou do edema tecidual, ou formação gradativa de hidrocefalia. Notadamente, nessa fase, destacase o rebaixamento do nível de consciência do paciente. Outros sintomas de hipertensão intracraniana começam a aparecer, como paralisia de VI nervo, paresias dimidiadas, liberação piramidal ou papiledema.

- A terceira e última fase é o coma propriamente dito, denotando compressão intensa das estruturas do tronco cerebral (formação reticular ascendente), pelo efeito de massa da lesão, e aparecimento de alterações cardiovasculares.

O conhecimento e o estudo dessas diferentes fases é importante para atuação médica, tão logo seja possível prever que um paciente possa evoluir para a fase seguinte e atuar o quanto antes, visando evitar seqüelas irreversíveis.

Os pacientes podem evoluir rapidamente nessas três fases ou permanecer somente na primeira ou na segunda, variando conforme o tamanho da lesão, o grau de compressão do IV ventrículo ou o sangramento intraventricular e com desenvolvimento de hidrocefalia ${ }^{1}$.

Em relação ao hematoma cerebelar espontâneo, reconhecem-se quatro formas de evolução ${ }^{1,2}$ (Quadro 2):

\begin{tabular}{lc}
\hline \multicolumn{2}{c}{ QUADRO 2 } \\
\multicolumn{1}{c}{ Evolução clínica das hemorragias ceberelares } \\
\hline Forma hiperaguda ou fulminante & $18 \%$ \\
Forma aguda & $14 \%$ \\
Forma subaguda & $28 \%$ \\
Forma benigna & $30 \%$ \\
\hline
\end{tabular}

- Forma hiperaguda ou fulminante - ocorre em cerca de $18 \%$ dos casos e é a forma mais grave e catastrófica; o paciente já chega em coma, com sinais de compressão de tronco evidentes (pupilas pontinas, alterações hemodinâmicas e respiratórias). Tem altíssima mortalidade e geralmente se associa a grandes hemorragias, com inundação de IV ventrículo e formação de hidrocefalia, edema exuberante do parênquima cerebelar e grande efeito expansivo e hipertensivo dentro do compartimento da fossa posterior.

- Forma aguda - a evolução para coma é mais lenta, em cerca de 24 a 48 horas após o icto, e o paciente pode chegar à emergência já torporoso ou sonolento, tendo tido os sinais premonitórios cerebelares. Representa 14\% dos casos, em média.

- Forma subaguda - nessa forma, a piora neurológica desenvolve-se tardiamente, entre o $7^{\circ} \mathrm{e} o$ $14^{\circ}$ dias, ocorre em cerca de $28-30 \%$ dos pacientes e tem bom prognóstico.

- Forma benigna - é responsável por $30 \%$ dos casos, inclui hematomas pequenos, sem efeito expansivo, geralmente com sintomas cerebelares estáveis durante todo o período, reabsorção do hematoma até cura do processo apenas com medidas conservadoras.

As hemorragias cerebelares são comumente localizadas nos hemisférios cerebelares, geralmente por ruptura de ramos da artéria cerebelar superior, e ocorrem principalmente em pacientes sabidamente hipertensos ${ }^{1,3}$. A localização mediana (vermiana) e a ocorrência de sangramentos em jovens devem ser investigadas quanto a outras etiologias (estrutural, por exemplo, malformações arteriovenosas ou tumores; infartos hemorrágicos por embolização central e discrasias sangüíneas). O trauma como causa de hemorragia cerebelar é raro, a não ser com outras condições prévias que a predisponham. 
Os infartos cerebelares são agrupados classicamente $\mathrm{em}^{4,5}$ :

- Territoriais - quando há acometimento de territórios específicos das artérias cerebelar superior (SCA), cerebelar póstero-inferior (PAICA) ou cerebelar ântero-inferior (AICA). São relacionados principalmente à doença vascular de grandes artérias.

- Não-territoriais - são infartos cerebelares pequenos, menores que $2 \mathrm{~cm}$ de diâmetro, freqüentemente encontrados em conjunto com os infartos territoriais, ou de aspecto múltiplo nos pacientes acometidos, e mais relacionados com doença de pequenos vasos.

Geralmente os infartos territoriais vêm combinados, com vários territórios arteriais comprometidos. A artéria mais comumente relacionada aos infartos cerebelares é a PAICA (cerca de $63 \%$ dos casos), seguidas pela AICA $(50 \%)$ e pela SCA $(23 \%)$, tanto nos subtipos territoriais quanto nos não-territoriais ${ }^{4}$, e a combinação desses dois subtipos também é bastante encontrada.

A etiologia principal nos infartos cerebelares é a aterosclerose e, em menor proporção, os acidentes embólicos e os casos de ectasia da artéria basilar ${ }^{4,5,6}$. Os fatores de risco vasculares são os mesmos para os infartos supratentoriais, como a hipertensão arterial, o diabetes, as dislipidemias e o tabagismo. Merecem atenção os casos de dissecção e trombose de vertebral e/ou de basilar, que constituem entidades à parte, devido à possibilidade de progressão dessas lesões vasculares ao longo de horas, a partir do quadro ictal, e seu pronto reconhecimento, para instituição de terapêutica, com auxílio de neurorradiologia intervencionista.

\section{DIAGNÓSTICO DE IMAGEM}

Os avanços no diagnóstico e na monitorização dos pacientes, na fase aguda, com a tomografia computadorizada do crânio (TC), trouxeram melhoria no manejo desses pacientes, que antes só tinham seu diagnóstico em exames post-mortem em muitos casos.

A TC sem injeção de contraste é o método de eleição, muito prático, disponível na maioria dos centros do mundo inteiro, realizado com rapidez, além de proporcionar grande definição no reconhecimento de sangue, na fase aguda da apresentação do processo, em que o ponto crucial é diferenciar quadros isquêmicos de hemorrágicos. A ressonância magnética (RM) revela, indubitavelmente, maior definição anatômica das estruturas da fossa posterior e está indicada no seguimento dos casos de infartos cerebelares, além de estudo mais aprofundado em imagens duvidosas que sugiram lesões estruturais vasculares ou tumorais ${ }^{3}$.

Em pacientes jovens ou com suspeita das lesões estruturais como causa dos acidentes vasculares cerebelares, a angiografia cerebral está indicada.

\section{TRATAME NTO}

Anteriormente à era da TC, o diagnóstico dos hematomas era difícil, apenas com a suspeita clínica e achados post-mortem; com a TC, as hemorragias passaram a ser tratadas clinicamente, e a monitorização do tamanho dos hematomas ou dos infartos em evolução facilitou o manejo da evolução dos casos.

As medidas gerais visam à monitorização da pressão arterial, aos parâmetros hemodinâmicos e cardiovasculares (alterados nas compressões do tronco cerebral) e à vigilância constante do nível de consciência e sua evolução, pois esse parâmetro indicará progressão das lesões.

TCs seriadas deverão ser realizadas, não havendo uma regra de intervalo entre elas; varia de acordo com o nível de consciência, o tamanho da lesão e a evolução do paciente. Geralmente, o rebaixamento do nível de consciência é o indicador da necessidade de monitorização neurorradiológica.

Hoje, o ponto mais discutido e alvo das diversas publicações sobre o assunto é estabelecer critérios para abordagem cirúrgica; a maioria dos autores concorda que o tamanho do hematoma e a compressão que exerce sobre o tronco cerebral são importantes nessa avaliação. Alguns trabalhos, com séries de casos estudados e prognóstico a longo prazo, estabeleceram que há indicação cirúrgica ${ }^{3,7}$ (Quadro 3):

\section{QUADRO 3}

Critérios para indicação cirúrgico em AVE cerebelar

Tamanho do hematoma $>3-4 \mathrm{~cm}$

Compressão do tronco cerebral e/ou cisternas da base

Deterioração do nível de consciência

Presença de hidrocefalia

- nos hematomas maiores que $3 \mathrm{~cm}$ ou $4 \mathrm{~cm}$, considerados passíveis de evacuação cirúrgica;

- se houver compressão do tronco cerebral, manifestada clinicamente pelo rebaixamento progressivo do nível de consciência; 
- na compressão progressiva da cisterna quadrigeminal, parâmetro mais recentemente estudado como índice de escolha para tratamento cirúrgico $^{8}$;

- a derivação ventriculoperitoneal está indicada se houver hidrocefalia, porém, isoladamente, ela pode levar à herniação ascendente, uma vez que o efeito de massa na fossa posterior permanece. Nesses casos, são necessárias evacuação/ descompressão da fossa posterior e derivação ou ventriculostomia ${ }^{7}$;

- rebaixamento progressivo do nível de consciência $($ Glasgow $<$ ou $=$ a 11$)$.

O acompanhamento clínico somente está reservado para hematomas pequenos, pacientes alertas e TC sem sinais de compressão do IV ventrículo ou do tronco ${ }^{3,9}$. De todos esses fatores, a variável mais importante considerada para a recuperação dos doentes, a longo prazo, é o nível de consciência, que se deteriora geralmente entre o segundo e o quarto dias, com pico no terceiro dia do icto ${ }^{10}$.

A craniotomia suboccipital aberta é a técnica mais utilizada para tratar os hematomas cerebelares. A abordagem para evacuação cirúrgica do hematoma é mediana ou paramediana. Quando necessário e indicado, procede-se a derivação do sistema ventricular concomitantemente.

Quanto aos infartos cerebelares, os mesmos critérios para decisão cirúrgica usados no manejo dos hematomas servem como parâmetros. Porém, há relatos do uso de terapia antiedema com manitol, hiperventilação e até dexametasona endovenosa, prévia aos procedimentos cirúrgicos. Alguns casos, tratados conservadoramente com essas medidas, melhoram sem necessidade de cirurgia $^{1,3,7}$. Alguns estudos demonstram que não há diferença entre tratamento clínico ou cirúrgico para pacientes alertas ou torporosos ${ }^{10}$, enquanto outros autores são categóricos em indicar terapia combinada de descompressão suboccipital do tecido infartado cerebelar, associada à derivação do sistema ventricular, para todos os pacientes com compressão do IV ventrículo e/ou do tronco cerebral, mesmo depois das medidas antiedema, visto que as técnicas cirúrgicas da descompressão e da derivação são simples e sem grandes sequielas para o paciente, quando comparadas com a lesão grave, e geralmente irreversíveis, como é a de uma compressão das estruturas do tronco cerebral ${ }^{11,12}$. Ensaios clínicos vêm sendo conduzidos com objetivo de estabelecer a melhor conduta ${ }^{13}$.

Recentemente, a drenagem de hematoma cerebelar por cirurgia estereotáxica vem sendo usada, principalmente em casos não complicados. A associação desse procedimento com o trombolítico local vem sendo estudada. Ainda não há evidências clínicas suficientes para estabelecer critérios dessas indicações.

\section{SUMMARY}

Cerebellar Hemorrhage and Infarction: Diagnostic and management The best therapeutic approach for cerebellar vascular diseases remains not well defined. There are not sufficient clinical trials to show us evidences about this topic. The recommendations for cerebellar stroke management are based on restrict experiences. There are also some controversies in surgical management.

\section{KEYWORDS}

Cerebellar infarction, cerebellar hemorrhage, diagnosis, treatment.

\section{Referências}

1. Heros RC. Cerebellar hemorrhage and infarction. Stroke, 13:106-109, 1982.

2. Galvão ACR. Hemorragias cerebelares espontâneas: aspectos clínicos e conduta terapêutica. Arq Bras Neurocirurg, 8:127-137, 1989.

3. Loftus CM. Management of cerebellar hematomas and infarcts. In: Welch KMA, Caplan LR et al. Primer on Cerebrovascular Diseases. Academic Press, San Diego, CA, USA, 1997.

4. Min WK, Kim YS et al. Atherothrombotic Cerebellar Infarction. Stroke, 30:2376-2381, 1999

5. Amarenco P, Lewy $\mathrm{C}$, Cohen $\mathrm{A}$ et al. Causes and mechanisms of territorial and non-territorial cerebellar infarcts in 115 consecutive patients. Stroke, 25:105-112, 1994.

6. Canaple S, Bogouslavsky J. Multiple large and small cerebellar infarcts. J Neurol Neurosurg Psychiatry, 66:739$745,1999$.

7. van der Hoop RG, Vermeulen M, Van Gijn J. Cerebellar hemorrhage: diagnosis and treatment. Surg Neurol, 29:610, 1988.

8 Taneda M, Hayakawa T, Mogami H. Primary cerebellar hemorrhage: quadrigeminal cistern obliteration on CT scans as a predictor of outcome. J Neurosurg, 67:545-552, 1987.

9. Kobayashi S, Sato A, Kageyama $Y$ et al. Treatment of hypertensive cerebellar hemorrhage - Surgical or conservative management? Neurosurgery, 34:246-251, 1994.

10. Jauss M, Krieger D, Hornig C, Schramm J, Busse O. Surgical and medical management of patients with massive cerebellar infarctions: results of the German-Austrian Cerebellar Infarction Study. J Neurol, 246:257-64, 1999

11. Chen H-J, Lee T-C, Wei C-P. Treatment of cerebellar infarction by descompressive suboccipital craniectomy. Stroke, 23:957-963, 1992.

12. Heros RC. Surgical treatment of cerebellar infarction. Stroke, 23:937-938, 1992.

13. Kreiger D, Busse O, Schramm J, Ferbert A. German-Austrian space occupying cerebellar infartion study (GASCIS): study design, methods, patients characteristics. J Neurol, 239:1835,1992

\section{Endereço para correspondência:}

Marcia Maiumi Fukujima

Disciplina de Neurologia - Escola Paulista de Medicina Unifesp

Rua Botucatu, 740

CEP 04023-900 - São Paulo, SP

Fone/Fax: (0XX11) 575-5240

E-mail:maiumi@sti.com.br 